[CONTRIBUTION FROM THE DEPARTMENT OF HOUSEHOT, SCIENCE OF THE UNIVERSITY OF CALIFORNIA.]

\title{
ON INULIN IN THE GLOBE ARTICHOKE.
}

By Ruth OKEY aNd ANNA W. Whlliams.

Received April 2\%, 1920.

The artichoke, Cyanara scolymus, sometimes called the bur or globe artichoke, is a common article of food, especially in California. Comparatively little has, however, been published as to its composition. In 1883 , an Italian investigator, de Regibus, ${ }^{1}$ found in the flowering head of this plant, a carbohydrate which he considered to be inulin. He also found that this carbohydrate disappeared as the seed matured, and that the reserve foodstuff finally stored was a fatty oil.

The composition of canned artichoke hearts, as determined by routine analysis, was reported by Wiley. ${ }^{2}$ His investigation, however, dealt primarily with the adulterants present, and with the economic value of the canned goods. The percentage of carbohydrate present was evidently estimated by difference, and no attempt made to determine its nature. Careful study of the abstract journals has failed to reveal any further reports on the composition of this plant.

The possibility that the artichoke might be a source of inulin suggested itself to the writers as a result of inquiries as to the nature of the white deposit, soluble in hot water, which separated in canned artichokes. This suggestion, combined with the local interest in the use of the artichoke as a food in California, and the meagerness of the available information, as to its composition led to the investigation which is reported here.

Material.-The artichokes used were purchased from a local market at various times between December, 1919, and April, 1920. The part of the plant usually sold as food, $i . e$., the immature flowering head, with about an inch and a half of the flower stalk, was used.

Preparation of Inulin.-In general, the technique used was that of Dean, ${ }^{3}$ modified as was found necessary. The artichokes were chopped with a sharp knife or put through a food chopper and, within 2 or 3 minutes after the beginning of the operation, thrown into an excess of boiling distilled water containing calcium carbonate. Boiling was continued for 40 minutes, or until the mass was soft and the separate pieces of material more or less thoroughly disintegrated. The extract was then poured off through a filter, replaced by a second portion of distilled water, and the extraction at boiling temperature repeated 3 or 4 times. The extracts obtained were treated exactly according to Dean's directions. ${ }^{3}$

${ }^{1}$ P. e. de Regibus, Giorn.d. R. Acad. medic., 45, 560 (1883); cf. H. Fischer, Cohn's "Biol. der Pflanzen," 8, 103 (1902).

"H.W. Wiley, Bur. Chem. Bull., 13, pt. 8, 128 \{1893\}.

A. L. Dean. Am. Chem. J., 32, 69 (1904). 
It was found desirable to subject the cut artichokes to the temperature of boiling water as quickly as possible, in order to prevent the blackening of the artichokes and the probable hydrolysis of the inulin by enzymes liberated in the bruising of the plant tissue.

Properties and Identification.--The substance obtained was easily precipitated as a white solid by $60 \%$ alcohol. It did not reduce Benedict's solution until after it had been hydrolyzed; after this process, however, an abundant precipitate was obtained, and Seliwanoff's reaction for levulose was positive. The hydrolyzed product was, moreover, levorotatory, and gave on treatment with phenylhydrazine hydrochloride and sodium acetate, in the usual manner, an osazone which had the crystalline form, and, after recrystallization from $50 \%$ alcohol, the melting point $\left(205-6^{\circ}\right)$ of dextrosazone. A sample was carefully purified, washed with absolute alcohol and absolute ether, and finally dried for several hours in a water oven at $100^{\circ}$. The rotation was then measured in aqueous solution, using a Schmidt and Häntsch saccharimeter. The value obtained was

$$
[\alpha]=-\frac{2.5 \times 100 \times 0.3468}{5.0 \times 0.5}=-34.68^{\circ} .
$$

These properties agreed with those of Dean's inulin. Moreover, it was shown that the specific rotation was very nearly identical with that of a similar solution of inulin which the writers had prepared in the same way from the tubers of the Jerusalem artichoke, Helianthus tuberosus. The substance was, therefore, considered to be inulin.

\section{General Analysis.}

Since Wiley's analyses ${ }^{2}$ had dealt with canned artichoke hearts only, a routine analysis of the material used seemed worth while, as did also an attempt to find the approximate percentages of the different types of carbohydrate present.

One large artichoke was put through a food chopper and portions for the various analyses weighed as rapidly as possible. Moisture, protein, ether extract, ash, and crude fiber were determined according to the official methods outlined in Bureau of Chemistry Bulletin No. 107 and the Journal of Official Agricultural Chemists. The figure for total carbohydrate was obtained by difference. Results are tabulated on p. 1695 . The figures given represent the results of at least 2 check determinations in each case.

s In general it was found that the inulin from the Jerusalem artichoke was precipitated more slowly by $60 \%$ alcohol than that from the globe artichoke. It seems probable, moreover, that there is a greater percentage of levulans of lower molecular peight in the Jerusalem artichoke than in the globe artichoke.

"Loc. cit. 


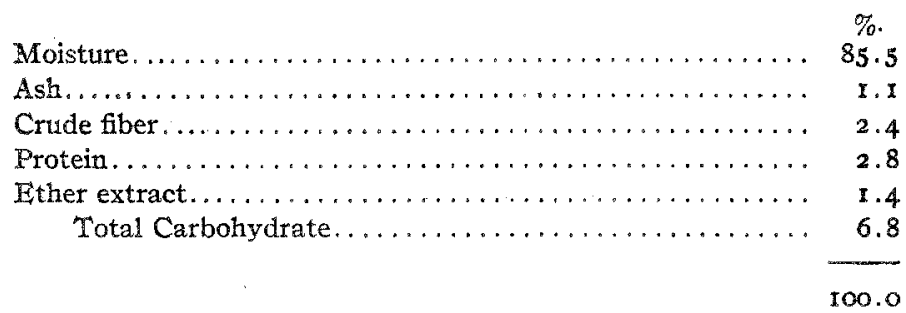

In order to get some idea of the percentage of this carbohydrate which consisted of inulin, fresh artichokes were taken, the blackened ends of the stems cut off, the flowering head and the stem quartered longitudinally. The samples so obtained were weighed rapidly and plunged, without loss of time, into boiling distilled water, and the boiling continued until the samples were soft. The water extract was poured off through a filter, replaced by a fresh portion of boiling distilled water, and the extraction repeated. After some 16 to 20 extractions had been made in this manner, the extracts were united, cooled, made up to definite volume (rooo cc. for a sample weighing about $40 \mathrm{~g}$.) and the reducing sugar determined by the modified Benedict ${ }^{1}$ colorimetric method, which has been shown by one of the writers ${ }^{2}$ to be applicable to the determination of levulose in the presence of small amounts of inulin. Hydrolyses of aliquot parts. of the extract were then carried out, using $1 \%$ hydrochloric acid at $100^{\circ}$, for lengths of time which had been shown ${ }^{2}$ to be sufficient for the complete hydrolysis of inulin. The resulting solutions were neutralized, made up to twice their original volumes and the levulose determined as before, the final time of heating the tubes being, in every case, 15 minutes, and the standard being pure glucose. Samples from different artichokes hydrolyzed separately gave results which checked in every case to $0.1 \%$.

The residues from the water extracts were then heated on a water bath with $\% \%$ hydrochloric acid for several hours, the resulting acid solutions poured through a filter, the residues washed thoroughly, and the washings added to the acid extracts. These were neutralized, made up to definite volume, and the reducing sugar in aliquot parts of the resulting solutions determined as before. The results obtained are listed below:

Reducing stugar (Corrected for color produced by inulin) $\ldots \ldots \ldots \ldots, 0.6$

Reducing sugar after hydrolysis of water extract............. 3.

Reducing sugar in residue from water soluble extract after hydrolysis , . $\quad 0.6$

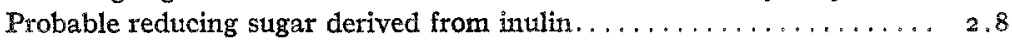

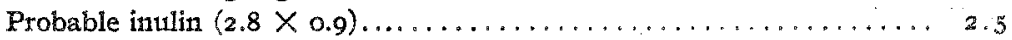

Total carbohydrate extracted by water and $1 \% \mathrm{HCl} \ldots \ldots \ldots \ldots \ldots, 4.2$

Difference from value, routine analysis $(6.8) \ldots \ldots \ldots \ldots \ldots \ldots, 6$

IS. R. Benedict and E. Osterberg, J. Biol. Chem, 34, 195 (19r8).

${ }^{2} \mathrm{R}$. Okey, ibid, 38, 33 (1919). 
There may be some question of the accuracy of results obtained by this method, because of the possible presence of other reducing substances, and the fact that the error is multiplied by such a large factor; but it seems fair, at least to consider that they represent proximate maximum values. The difference between the values for "Total carbohydrates extracted by water and I\% HCl" and for "total carbohydrates by difference" would indicate the presence of hemicellulose-like substances. Moreover, the structure of the artichoke makes this seems the more probable.

It has been shown ${ }^{1}$ that the human digestive tract secretes no enzymes capable of digesting either hemicellulose or inulin, and there is some evidence $^{2}$ that inulin is not utilized by the animal body.

There is some reason to doubt, therefore, whether the larger part of the carbohydrate of the globe artichoke is in a form that can be utilized by the human organism.

BERKELEY, CALIF.

[Contribution from the Department of Chemistry, Columbia University, No. 336.]

\section{THE SPECIFIC ROTATION OF FRUCTOSE.}

BY WARREN C. VOSBURGH. ${ }^{3}$

Received May 21, 1920.

In the course of an investigation of the determination of the amount of sucrose and invert sugar in their mixtures it was found that considerable disagreement exists in the literature as to the specific rotation of fructose. For example the specific rotation for a concentration of $5 \mathrm{~g}$. per Ioo $\mathrm{cc}$. (4.93\% by weight) according to several observers is as follows.

$\begin{array}{cc}{[\alpha]_{\mathbf{D}}^{20}} & {[\alpha]_{\mathbf{D}}^{25}} \\ 89.64 & 86.84 \\ 89.40 & 86.04 \\ 92.45 & 89.65 \\ 91.0^{\circ} & 88.0\end{array}$

Jungfleisch and Grimbert ${ }^{a}$

Honig and Jesser ${ }^{b}$

$\mathrm{Ost}^{d}$

92.45

88.0

Nelson and Beegle $f$

${ }^{a}$ Jungfleisch and Grimbert, $[\alpha]_{\mathrm{D}}^{t}=-($ ror. $38-0.56 t+0.108(c-10))$, Compt. rend., 107, 390 (1888).

${ }^{3}$ Honig and Jesser, $[\alpha]_{\mathrm{D}}^{t}=-(88.13-0.2385 p+0.6714(t-20))$, J. Deut. Zucker. ind., 38 , г028 (I888).

- Using the temperature coefficient of Jungfleisch and Grimbert.

${ }^{a}$ Ost, $[\alpha]_{\mathrm{D}}^{20}=-(91.90+0.1 \mathrm{II} p)$, Ber., 24, 1636 (1891).

${ }^{\circ}$ Interpolated between $15^{\circ}$ and $25^{\circ}$.

$f$ Nelson and Beegle, This Journal, 4I, 559 (1919).

1 Cf. M. D. Swartz, Trans, Conn. Acad. Sci., 16, 297 (I9I1), for a review of this literature.

' R. Okey, J, Biol. Chem., 39, I49 (1919).

National Research Fellow. 